\title{
Stochastic Effects for the Reaction-Duffing Equation with Wick-Type Product
}

\author{
Jin Hyuk Choi, ${ }^{1}$ SeungGwan Lee, ${ }^{1}$ and Hyunsoo Kim ${ }^{2}$ \\ ${ }^{1}$ Humanitas College, Kyung Hee University, Yongin 446-701, Republic of Korea \\ ${ }^{2}$ Department of Mathematics, Sungkyunkwan University, Suwon 440-746, Republic of Korea \\ Correspondence should be addressed to Hyunsoo Kim; hskiminkorea@gmail.com
}

Received 8 April 2016; Accepted 9 June 2016

Academic Editor: Antonio Scarfone

Copyright (c) 2016 Jin Hyuk Choi et al. This is an open access article distributed under the Creative Commons Attribution License, which permits unrestricted use, distribution, and reproduction in any medium, provided the original work is properly cited.

\begin{abstract}
We construct new explicit solutions of the Wick-type stochastic reaction-Duffing equation arising from mathematical physics with the help of the white noise theory and the system technique. Based on these exact solutions, we also discuss the influences of stochastic effects for dynamical behaviors according to functions $h_{1}(t), h_{2}(t)$, and Brownian motion $B(t)$ which are the solitary wave group velocities.
\end{abstract}

\section{Introduction}

We consider the following generalized Wick-type stochastic reaction-Duffing equation with the variable coefficients and the white noise environment:

$$
\begin{aligned}
U_{t}+ & H_{1}(t) \diamond U_{\mathbf{x}} \\
& +H_{2}(t) \diamond\left(A U_{\mathbf{x x}}+C U^{\diamond 3}+E U^{\diamond 2}+D U\right)=0,
\end{aligned}
$$

where $\diamond$ is the Wick product on the Hida distribution space $S\left(\mathbb{R}^{d}\right)^{*}$ such as the white noise functional space and $H_{1}(t)=$ $h_{1}(t)+k_{1} W(t)$ and $H_{2}(t)=h_{2}(t)+k_{1} W(t)[1,2]$. Here $k_{1}$ and $k_{2}$ are constants, $W(t)$ is the Gaussian white noise, $h_{1}(t)$ and $h_{2}(t)$ are two arbitrary functions, and $A, C, E$, and $D$ are real constants. The concern of the Wick-type stochastic equations is very common because many authors discussed the Wick-type stochastic equations with variable coefficients and fractional derivatives and so forth [3-6].

Constructing new explicit solutions of nonlinear evolution equations arising from the field of mathematical physics and engineering is an important topic. However, solving the nonlinear evolution equations is much more difficult than solving the linear ones. Most of physical phenomena coming from the fields of mathematical physics and engineering can be described by the closed-form solutions of nonlinear partial differential equations. So, a great deal of attention has been paid towards both exact and numerical solutions of these equations. Thus we need many efforts for studying mathematical algorithms for determining the exact solutions of nonlinear evolution equations. Recently, there are many effective and powerful methods for obtaining exact solutions to know physical phenomena in mathematical physics and engineering and also we need the study for the development of some techniques, for example, the stochastic linearization method [7], the wavelet-based method [8], the quasi-static method [9], differential transform method [10, 11], factorization method [12], extended Jacobi elliptic function expansion method [13, 14], tanh-expansion method $[15,16],\left(G^{\prime} / G\right)$ expansion method $[17,18]$, and Kudryashov method [1922]. Moreover, through these methods, we know that explicit solutions of nonlinear partial differential equations can reveal the internal mechanism of physical phenomena. As well as physical importance, the closed-form solutions of nonlinear partial differential equations can assist the numerical solvers to compare the correctness of their results and can help them in the stability analysis.

We would like to use the system technique that contains exponential function based on suitable choices of parameters. We show that the system technique has its validity and potential for obtaining new explicit solutions of (1), which contain kink-type motions and soliton-type motions of physical phenomena and other new types of exact solutions. 


\section{Descriptions of the Wick-Type Product and Algorithm for Finding Solutions}

The space $(S)_{-1}^{n}$ consists of all formal expansions $F(\xi)=$ $\sum_{\alpha} b_{\alpha} H_{\alpha}(\xi)$ with $b_{\alpha} \in \mathbb{R}^{n}$ and $\xi=\left(\xi_{0}, \ldots, \xi_{m}\right) \in(S(\mathbb{R}))^{*}$. Here $(S(\mathbb{R}))^{*}$ is the Hida distribution space on $\mathbb{R}$. The Wick product $f \diamond F$ of two elements $f=\sum_{\alpha} a_{\alpha} H_{\alpha}, F=\sum_{\beta} b_{\beta} H_{\beta} \in$ $(S)_{-1}^{n}$ with $a_{\alpha}, b_{\beta} \in \mathbb{R}^{n}$ is defined by

$$
f \diamond F=\sum_{\alpha, \beta}\left(a_{\alpha}, b_{\beta}\right) H_{\alpha+\beta} .
$$

For $F=\sum_{\alpha} b_{\alpha} H_{\alpha} \in(S)_{-1}^{N}$, with $b_{\alpha} \in \mathbb{R}^{\mathbb{N}}$, the Hermite transformation of $F$, denoted by $\widetilde{F}(\mathrm{z})$, is defined by

$$
\widetilde{F}(\mathrm{z})=\sum b_{\alpha} \mathrm{z}^{\alpha} \in \mathbb{C}^{\mathbb{N}} \quad(\text { when convergent }),
$$

where $z=\left(z_{1}, z_{2}, \ldots\right) \in \mathbb{C}^{\mathbb{N}}$ (the set of all sequences of complex numbers) and $z^{\alpha}=z_{1}^{\alpha_{1}} z_{2}^{\alpha_{2}} \cdots z_{n}^{\alpha_{n}} \cdots$ for $\alpha=\left(\alpha_{1}\right.$, $\left.\alpha_{2}, \ldots\right) \in\left(\mathbb{N}_{0}\right)_{c}^{\mathbb{N}}$, where $\alpha_{i} \in \mathbb{N}_{0}$ and $z_{j}^{0}=1$.

For $F, G \in(S)_{-1}^{n}$, by this definition, we have

$$
\widetilde{F \diamond G}(\mathrm{z})=\widetilde{F}(\mathrm{z}) \cdot \widetilde{G}(\mathrm{z}),
$$

for all $z$ such that $\widetilde{F}(z)$ and $\widetilde{G}(z)$ exist. The product on the right hand side of the above formula is the complex bilinear product between two elements of $\mathbb{C}^{n}$ defined by $\left(z_{1}^{1}, \ldots, z_{n}^{1}\right)$. $\left(\mathrm{z}_{1}^{2}, \ldots, \mathrm{z}_{n}^{2}\right)=\sum_{k=1}^{n} \mathrm{z}_{k}^{1} \mathrm{z}_{k}^{2}$, where $\mathrm{z}_{k}^{i} \in \mathbb{C}$.

Let $X=\sum_{\alpha} a_{\alpha} H_{\alpha} \in(S)_{-1}^{\mathbb{N}}$. Then the vector $c_{0}=\widetilde{X}(0) \epsilon$ $\mathbb{R}^{n}$ is called the generalized expectation of $X$ and is denoted by $E(X)$. Suppose that $g: U \rightarrow \mathbb{C}^{m}$ is an analytic function, where $U$ is a neighborhood of $c_{0}:=E(X)$. Assume that the Taylor series of $g$ around $c_{0}$ have coefficients in $\mathbb{R}^{m}$. Then the Wick version $g^{\diamond}(X)=\mathscr{H}^{-1}(g \circ \widetilde{X})$ is an element of $(S)_{-1}^{m}$. In other words, if $g$ has the power series expansion $g(z)=$ $\sum a_{\alpha}\left(z-c_{0}\right)^{\alpha}$ with $a_{\alpha} \in \mathbb{R}^{n}$, then $g^{\diamond}(X)=\sum a_{\alpha}\left(X-c_{0}\right)^{\diamond \alpha} \in$ $(S)_{-1}^{m}$.

The Wick exponent of $X \in(S)_{-1}^{n}$ is defined by $\exp ^{\diamond}\{X\}=$ $\sum_{n=0}^{\infty} X^{\diamond n} / n$ !. With the Hermite transformation, the Wick exponent shows the same algebraic properties as the usual one. For example, $\exp ^{\diamond}\{X+Y\}=\exp ^{\diamond}\{X\} \diamond \exp ^{\diamond}\{Y\}$.

We next outline the main processes of the compatibility method for the Wick-type stochastic partial differential equations. Suppose that modeling considerations lead us to consider the stochastic partial differential equation expressed formally as

$$
\mathscr{P}\left(t, \mathbf{x}, \partial_{t}, \nabla_{\mathbf{x}}, U, \varsigma\right)=0,
$$

where $\mathscr{P}$ is a polynomial, in which the highest order derivatives and nonlinear terms are involved, and $U=U(\mathbf{x}, t, \varsigma)$ is the unknown (generalized) stochastic process and where the operators $\partial_{t}=\partial / \partial t, \nabla_{\mathbf{x}}=\left(\partial / \partial \mathbf{x}_{1}, \ldots, \partial / \partial \mathbf{x}_{d}\right)$ when $\mathbf{x}=$ $\left(\mathbf{x}_{1}, \ldots, \mathbf{x}_{d}\right) \in \mathbb{R}^{d}$.

With the aid of the Hermite transformation, we transform the Wick-type equation

$$
\mathscr{P}^{\diamond}\left(t, \mathbf{x}, \partial_{t}, \nabla_{\mathbf{x}}, U, \varsigma\right)=0
$$

into an ordinary product equation with variable coefficients

$$
\widetilde{\mathscr{P}}\left(t, \mathbf{x}, \partial_{t}, \nabla_{\mathbf{x}}, \widetilde{U}, \mathrm{z}_{1}, \mathrm{z}_{2}, \ldots\right)=0,
$$

where $\widetilde{U}=\mathscr{H}(\mathcal{U})$ is the Hermite transform of $U$ and $z_{1}$, $\mathrm{z}_{2}, \ldots$ are complex numbers.

Suppose that we can get exact solutions $u=\widetilde{U}(t, \mathbf{x}, \mathbf{z})$ of (7) for each $z \in \mathbb{K}_{q}(r)$, where $\mathbb{K}_{q}(r)=\left\{z=\left(z_{1}, z_{2}, \ldots\right) \in\right.$ $\mathbb{C}^{\mathbb{N}}$ and $\left.\sum_{\alpha \neq 0}\left|z^{\alpha}\right|^{2}(2 \mathbb{N})^{q \alpha}<r^{2}\right\}$ for some $q, r$. For a start, we would like to introduce the process for obtaining exact solutions of (7) as follows.

Combining the variables $\mathbf{x}$ and $t$ into one traveling wave variable $\eta$, we suppose that traveling wave transformation,

$$
u(\mathbf{x}, t, \mathrm{z})=\mathrm{u}(\eta), \quad \eta=k \mathbf{x}-\omega(t, \mathrm{z})
$$

permits us to reduce (7) to an ordinary differential equation, for $\mathrm{u}=\mathrm{u}(\eta)$,

$$
Q\left(u, u^{\prime}, u^{\prime \prime}, u^{\prime \prime \prime}, \ldots\right)=0
$$

where $\mathrm{u}^{\prime}=d \mathrm{u} / d \eta, \mathrm{u}^{\prime \prime}=d^{2} \mathrm{u} / d \eta^{2}, \mathrm{u}^{\prime \prime \prime}=d^{3} \mathrm{u} / d \eta^{3}, \ldots$

We can get the $m$-order pole solution of (9) that the positive integer $m$ can be determined by considering the homogeneous balance property between the highest order derivatives of linear term and nonlinear term appearing in (9). Suppose that exact solution of (9) can be expressed by a polynomial in $(\mathrm{F}(\eta) / \mathrm{G}(\eta))$ as follows:

$$
\begin{aligned}
\mathrm{u}(\eta)= & A_{m}\left(\frac{\mathrm{F}(\eta)}{\mathrm{G}(\eta)}\right)^{m}+A_{m-1}\left(\frac{\mathrm{F}(\eta)}{\mathrm{G}(\eta)}\right)^{m-1}+\cdots \\
& +A_{1}\left(\frac{\mathrm{F}(\eta)}{\mathrm{G}(\eta)}\right)+A_{0}
\end{aligned}
$$

where $F(\eta)$ and $G(\eta)$ are satisfying the system

$$
\begin{aligned}
& \mathrm{F}^{\prime}(\eta)=p \mathrm{~F}(\eta), \\
& \mathrm{G}^{\prime}(\eta)=p \mathrm{~F}(\eta)+q \mathrm{G}(\eta),
\end{aligned}
$$

where $p$ and $q$ are arbitrary constants, and we will call it the system, and $A_{m}, A_{m-1}, \ldots, A_{1}, A_{0}, k$ and $\omega$ will be determined later with $A_{m} \neq 0$.

System (11) gives us the following function:

$$
\left(\frac{\mathrm{F}(\eta)}{\mathrm{G}(\eta)}\right)=\frac{p-q}{p-q \exp \{-(p-q) \eta\}},
$$

where $p$ and $q$ are arbitrary constants with $p \neq q$ in Remark 1 .

By substituting (10) into (9), collecting all terms with the same order of $(F(\eta) / G(\eta))$ together, the left-hand side of (9) is converted into another polynomial in $(\mathrm{F}(\eta) / \mathrm{G}(\eta))$ using derivatives in Remark 2. Equating each coefficient of this polynomial to zero, we have a set of algebraic equations for $A_{m}, A_{m-1}, \ldots, A_{1}, A_{0}, k$, and $\omega$.

Assuming that the coefficients $A_{m}, A_{m-1}, \ldots, A_{1}, A_{0}$ and traveling wave coefficients $k, \omega$ can be obtained by solving the algebraic equations in the above, since the general solutions 
of system (11) have been known for us, then by substituting $A_{m}, A_{m-1}, \ldots, A_{1}, A_{0}, k$ and $\omega$ into (10), we have more new explicit solutions of (9) by traveling wave transformation (8).

Finally, we would end up with the explicit expressions for some undetermined coefficients and then we can obtain explicit solutions of (7) by substituting them into $u=$ $\widetilde{U}(t, \mathbf{x}, \mathbf{z})$. Moreover, under certain conditions, we take the inverse Hermite transformation $U=\mathscr{H}^{-1} u \in(S)_{-1}$ and thereby can obtain a Wick-type solution $U$ of (6) [23].

Remark 1. The integrability conditions of the system, given by (11), have been extensively discussed in the following literature, and so the relations among the coefficients of the system involve two constraints as follows: $p \neq q$ and $p=q$. Firstly, we consider the solution of (11) for $p \neq q$. Assume $\mathrm{F}(0)=0$ and $\mathrm{G}(0)=0$ without loss of generality. From the first part of (11), the solution $\mathrm{F}(\eta)$ is given by

$$
\mathrm{F}(\eta)=e^{p \eta}
$$

Substituting (13) into the second equation of (11), we get a differential equation

$$
\mathrm{G}^{\prime}(\eta)-q \mathrm{G}(\eta)=e^{p \eta}
$$

Then, we can find the solution of (14) as follows:

$$
\mathrm{G}(\eta)=e^{q \eta}\left[\frac{p}{p-q} e^{-(p-q) \eta}-\frac{q}{p-q}\right]
$$

Combining (13) and (15), we obtain the following function:

$$
\left(\frac{\mathrm{F}(\eta)}{\mathrm{G}(\eta)}\right)=\frac{p-q}{p-q \exp \{-(p-q) \eta\}}
$$

where $p$ and $q$ are arbitrary nonzero constants with $p \neq q$.

On the other hand, when $p=q$, we have another function:

$$
\left(\frac{\mathrm{F}(\eta)}{\mathrm{G}(\eta)}\right)=\frac{1}{\eta+1} .
$$

Remark 2. The following derivatives are very useful for equating the expressions at the same degrees of $(F / G)$ to zero in (9): $(\mathrm{F} / \mathrm{G})^{\prime}=(p-q)(\mathrm{F} / \mathrm{G})-p(\mathrm{~F} / \mathrm{G})^{2},(\mathrm{~F} / \mathrm{G})^{\prime \prime}=(p-$ $q)^{2}(\mathrm{~F} / \mathrm{G})-3 p(p-q)(\mathrm{F} / \mathrm{G})^{2}+2 p^{2}(\mathrm{~F} / \mathrm{G})^{3},(\mathrm{~F} / \mathrm{G})^{\prime \prime \prime}=(p-$ $q)^{3}(\mathrm{~F} / \mathrm{G})-6 p(p-q)(\mathrm{F} / \mathrm{G})^{2}+2 p^{2}(p-q+1)(\mathrm{F} / \mathrm{G})^{3}-6 p^{3}(\mathrm{~F} / \mathrm{G})^{4}$, and so on.

\section{Wick-Type Explicit Solutions of (1)}

Taking the Hermit transformation in (1), we obtain the following equation:

$$
\begin{aligned}
& \widetilde{U}_{t}(t, \mathbf{x}, \mathbf{z})+\widetilde{H}_{1}(t, \mathbf{z}) \widetilde{U}_{\mathbf{x}}(t, \mathbf{x}, \mathbf{z})+\widetilde{H}_{2}(t, \mathbf{z}) \\
& \cdot\left\{A \widetilde{U}_{\mathbf{x x}}(t, \mathbf{x}, \mathbf{z})+C \widetilde{U}^{3}(t, \mathbf{x}, \mathbf{z})+E \widetilde{U}^{2}(t, \mathbf{x}, \mathbf{z})\right. \\
& +D \widetilde{U}(t, \mathbf{x}, \mathbf{z})\}=0,
\end{aligned}
$$

where $\mathbf{z}=\left(\mathrm{z}_{1}, \mathrm{z}_{2}, \ldots\right) \in\left(\mathbb{C}^{n}\right)_{c}$ is a vector parameter.

To simplify (18), we take traveling wave transformation

$$
\widetilde{U}(t, \mathbf{x}, \mathrm{z})=u(t, \mathbf{x}, \mathrm{z})=\mathrm{u}(\eta), \quad \eta=k \mathbf{x}-\omega(t, \mathrm{z}),
$$

and $\widetilde{H}_{1}(t, z)=H_{1}(t, z)$ and $\widetilde{H}_{2}(t, z)=H_{2}(t, z)$. Then we have

$$
\begin{aligned}
& \left(\omega^{\prime}(t, \mathrm{z})+k H_{1}(t, \mathrm{z})\right) \mathrm{u}^{\prime} \\
& \quad+H_{2}(t, \mathrm{z})\left\{A k^{2} \mathrm{u}^{\prime \prime}+C \mathrm{u}^{3}+E \mathrm{u}^{2}+D \mathrm{u}\right\}=0
\end{aligned}
$$

where $\mathrm{u}^{\prime}=d \mathrm{u} / d \eta, \mathrm{u}^{\prime \prime}=d^{2} \mathrm{u} / d \eta^{2}$, and $\omega^{\prime}=d \omega / d t$.

Now, let us find exact solutions of (20) by the system technique. It can be easily seen that (20) has the first-order pole solution by the balancing property. Suppose that explicit solution of (20) can be expressed in the form

$$
\mathrm{u}(\eta)=A_{0}(t, \mathrm{z})+A_{1}(t, \mathrm{z})\left(\frac{\mathrm{F}(\eta)}{\mathrm{G}(\eta)}\right)
$$

where $(F(\eta) / G(\eta))$ is given by $(12)$ and $F(\eta)$ and $G(\eta)$ satisfy system (11).

By taking expression (21) and using Remark 2, we can obtain the derivatives $u^{\prime}$ and $u^{\prime \prime}$ expressed via the function $(\mathrm{F}(\eta) / \mathrm{G}(\eta))$. By substituting $\mathrm{u}, \mathrm{u}^{\prime}$, and $\mathrm{u}^{\prime \prime}$ into (20) and equating to zero the expressions with the same degrees of $(\mathrm{F}(\eta) / \mathrm{G}(\eta))$, we can obtain the set of algebraic equations with respect to the unknowns $A_{1}(t, \mathrm{z}), A_{0}(t, \mathrm{z}), k$, and $\omega(t, \mathrm{z})$ and, by solving the algebraic system by the help of Maple, we obtain the following five explicit solutions of (18) with traveling wave transformation (19).

Let $K_{1}=2 C D+E^{2}+\sqrt{E^{4}-4 C E^{2} D}$ and $K_{2}=2 C D+$ $E^{2}-\sqrt{E^{4}-4 C E^{2} D}$ be constants to reduce the forms of explicit solutions of (18). The first exact solution of (18) can be written as

$$
\widetilde{U}_{1}(\mathbf{x}, t, \mathbf{z})=\frac{\left(K_{1}-2 D C\right) p}{E}\left[\frac{1}{p-q \exp \left\{-(p-q)\left( \pm\left(\sqrt{C A K_{1}} / 2 C A(p-q)\right) \mathbf{x}-\omega(t, \mathbf{z})\right)\right\}}\right]
$$

where $\omega(t, z)=\left(1 /(p-q)^{2}\right) \int_{0}^{t}\left\{\left(\mp\left(\sqrt{C A K_{1}} / 2 C A\right)(p \pm q)-(p-\right.\right.$ q)D $\left.) H_{1}(\tau, \mathbf{z})+\left(K_{1} q / 2 C\right) H_{2}(\tau, \mathbf{z})\right\} d \tau$.
The second exact solution of (18) can be expressed as 


$$
\widetilde{U}_{2}(\mathbf{x}, t, \mathbf{z})=\frac{\left(K_{2}-2 D C\right) p}{E}\left[\frac{1}{p-q \exp \left\{-(p-q)\left( \pm\left(\sqrt{C A K_{2}} / 2 C A(p-q)\right) \mathbf{x}-\omega(t, \mathbf{z})\right)\right\}}\right],
$$

where $\omega(t, \mathrm{z})=\left(1 /(p-q)^{2}\right) \int_{0}^{t}\left\{\left(\mp\left(\sqrt{C A K_{2}} / 2 C A\right)(p \pm q)-(p-\right.\right.$ q)D) $\left.H_{1}(\tau, \mathrm{z})+\left(K_{2} q / 2 C\right) H_{2}(\tau, \mathrm{z})\right\} d \tau$.
The third exact solution of (18) is given by

$$
\begin{aligned}
\widetilde{U}_{3}(\mathbf{x}, t, \mathrm{z})= & -\frac{E \mp \sqrt{E^{2}-4 C D}}{2 C}+\frac{\left(12 C D-2 E^{2}-\left(E \mp \sqrt{E^{2}-4 C D}\right)\right) p}{C E\left(2-3\left(E \mp \sqrt{E^{2}-4 C D}\right)\right)} \\
& \times\left[\frac{1}{p-q \exp \left\{-(p-q)\left( \pm(1 /(p-q)) \sqrt{-\left(E^{2}-4 C D\right) / 2 C A} \mathbf{x}-\omega(t, \mathrm{z})\right)\right\}}\right],
\end{aligned}
$$

where $\omega(t, \mathrm{z}) \quad=\quad\left(1 / C(p-q)^{2}\right) \int_{0}^{t}\{\mp(p \quad \pm \quad$ The fourth exact solution of (18) is q) $C \sqrt{-\left(E^{2}-4 C D\right) / 2 C A} H_{1}(\tau, \mathrm{z})-\left((1 / 2) E\left(E-\sqrt{E^{2}-4 C D}\right)(p\right.$ $\left.\left.-q)-2 p C D+E^{2} q-2 q C D\right) H_{2}(\tau, z)\right\} d \tau$.

$$
\begin{aligned}
\widetilde{U}_{4}(\mathbf{x}, t, z)= & -\frac{E \mp \sqrt{E^{2}-4 C D}+\frac{2\left(3 C D-E^{2}+\sqrt{E^{4} \mp 4 C E^{2} D}\right) p}{C\left(E-3 \sqrt{E^{2}-4 C D}\right)}}{2 C} \\
& \times\left[\frac{1}{p-q \exp \left\{-(p-q)\left( \pm(1 /(p-q)) \sqrt{-K_{1} / 4 C A} \mathbf{x}-\omega(t, z)\right)\right\}}\right],
\end{aligned}
$$

where $\omega(t, z)=\left(1 /(p-q)^{2}\right) \int_{0}^{t}\left\{\mp(p-q) \sqrt{-K_{1} / 4 C A} H_{1}(\tau, z)+\right.$

The last exact solution of (18) is $\left.\left(D(2 p-q)-E\left(E \mp \sqrt{E^{2}-4 C A}\right) p / 2 C\right) H_{2}(\tau, z)\right\} d \tau$.

$$
\begin{aligned}
\widetilde{U}_{5}(\mathbf{x}, t, \mathrm{z})= & -\frac{E \mp \sqrt{E^{2}-4 C D}}{2 C}+\frac{2\left(3 C D-E^{2}+\sqrt{E^{4} \mp 4 C E^{2} D}\right) p}{C\left(E-3 \sqrt{E^{2}-4 C D}\right)} \\
& \times\left[\frac{1}{p-q \exp \left\{-(p-q)\left( \pm(1 /(p-q)) \sqrt{-K_{2} / 4 C A} \mathbf{x}-\omega(t, z)\right)\right\}}\right],
\end{aligned}
$$

where $\omega(t, \mathrm{z})=\left(1 /(p-q)^{2}\right) \int_{0}^{t}\left\{\mp(p-q) \sqrt{-K_{2} / 4 C A} H_{1}(\tau, \mathrm{z})+\right.$ $\left.\left(D(2 p-q)-E\left(E \mp \sqrt{E^{2}-4 C A}\right) p / 2 C\right) H_{2}(\tau, z)\right\} d \tau$.

In order to obtain white functional solutions for (1), we use the Hermit transformation and Theorem 4.1.1 in [23]. The property of generalized exponential functions yields that there exists a bounded open set $\mathbf{G} \subset \mathbb{R} \times \mathbb{R}_{+}, k<\infty, r>0$ such that the exact solutions $\widetilde{U}(\mathbf{x}, t, \mathbf{z})$ of (18) are uniformly bounded for $(\mathbf{x}, t, \mathbf{z}) \in \mathbf{G} \times \mathbb{K}_{q}(r)$, continuous with respect to $(\mathbf{x}, t) \in \mathbf{G}$ and analytic with respect to $z \in \mathbb{K}_{k}(r)$, for all $(\mathbf{x}, t) \in \mathbf{G}$ [24]. There exists $\Psi(\mathbf{x}, t, \mathbf{z}) \in(S)_{-1}$ such that $\widetilde{U}(\mathbf{x}, t, \mathbf{z})=(\widetilde{U}(t, \mathbf{x}))(\mathbf{z})$ for all $(t, \mathbf{x}, \mathbf{z}) \in \mathbf{G} \times \mathbb{K}_{q}(r)$ and $U(\mathbf{x}, t)$ solves (in the strong sense in $\left.(S)_{-1}\right)(1)$ in $(S)_{-1}$. Hence, by applying the inverse Hermite transformation to solutions (22)-(26), we can obtain the following Wick-type solutions of (1). Based on solutions (22) and (23), we get the following Wick-type solutions as follows:

$$
U_{1}(\mathbf{x}, t)=\frac{\left(K_{1}-2 D C\right) p}{E}\left[\frac{1}{p-q \exp ^{\diamond}\left\{-(p-q)\left( \pm\left(\sqrt{C A K_{1}} / 2 C A(p-q)\right) \mathbf{x}-\omega(t)\right)\right\}}\right]
$$


where $\omega(t)=\left(1 /(p-q)^{2}\right) \int_{0}^{t}\left\{\left(\mp\left(\sqrt{C A K_{1}} / 2 C A\right)(p \pm q)-(p-\right.\right.$ q)D) $\left.H_{1}(\tau)+\left(K_{1} q / 2 C\right) H_{2}(\tau)\right\} d \tau$, and

$$
U_{2}(\mathbf{x}, t)=\frac{\left(K_{2}-2 D C\right) p}{E}\left[\frac{1}{p-q \exp ^{\diamond}\left\{-(p-q)\left( \pm\left(\sqrt{C A K_{2}} / 2 C A(p-q)\right) \mathbf{x}-\omega(t)\right)\right\}}\right],
$$

where $\omega(t)=\left(1 /(p-q)^{2}\right) \int_{0}^{t}\left\{\left(\mp\left(\sqrt{C A K_{2}} / 2 C A\right)(p \pm q)-(p-\right.\right.$ q)D) $\left.H_{1}(\tau)+\left(K_{2} q / 2 C\right) H_{2}(\tau)\right\} d \tau$.
From (24), we obtain the following Wick-type solution of (1):

$$
\begin{aligned}
U_{3}(\mathbf{x}, t)= & -\frac{E \mp \sqrt{E^{2}-4 C D}}{2 C}+\frac{\left(12 C D-2 E^{2}-\left(E \mp \sqrt{E^{2}-4 C D}\right)\right) p}{C E\left(2-3\left(E \mp \sqrt{E^{2}-4 C D}\right)\right)} \\
& \times\left[\frac{1}{p-q \exp ^{\diamond}\left\{-(p-q)\left( \pm(1 /(p-q)) \sqrt{-\left(E^{2}-4 C D\right) / 2 C A} \mathbf{x}-\omega(t)\right)\right\}}\right],
\end{aligned}
$$

q) $C \sqrt{-\left(E^{2}-4 C D\right) / 2 C A} H_{1}(\tau)+\left(2 p C D-E^{2} q+2 q C D-\right.$ $\left.\left.(1 / 2) E\left(E-\sqrt{E^{2}-4 C D}\right)(p-q)\right) H_{2}(\tau)\right\} d \tau$.

$$
\begin{aligned}
U_{4}(\mathbf{x}, t)= & -\frac{E \mp \sqrt{E^{2}-4 C D}}{2 C}+\frac{2\left(3 C D-E^{2}+\sqrt{E^{4} \mp 4 C E^{2} D}\right) p}{C\left(E-3 \sqrt{E^{2}-4 C D}\right)} \\
& \times\left[\frac{1}{p-q \exp ^{\diamond}\left\{-(p-q)\left( \pm(1 /(p-q)) \sqrt{-K_{1} / 4 C A} \mathbf{x}-\omega(t)\right)\right\}}\right],
\end{aligned}
$$

where $\omega(t)=\left(1 /(p-q)^{2}\right) \int_{0}^{t}\left\{\mp(p-q) \sqrt{-K_{1} / 4 C A} H_{1}(\tau)+\right.$ $\left.\left(D(2 p-q)-E\left(E \mp \sqrt{E^{2}-4 C A}\right) p / 2 C\right) H_{2}(\tau)\right\} d \tau$, and

$$
\begin{aligned}
U_{5}(\mathbf{x}, t)= & -\frac{E \mp \sqrt{E^{2}-4 C D}}{2 C}+\frac{2\left(3 C D-E^{2}+\sqrt{E^{4} \mp 4 C E^{2} D}\right) p}{C\left(E-3 \sqrt{E^{2}-4 C D}\right)} \\
& \times\left[\frac{1}{p-q \exp ^{\diamond}\left\{-(p-q)\left( \pm(1 /(p-q)) \sqrt{-K_{2} / 4 C A} \mathbf{x}-\omega(t)\right)\right\}}\right],
\end{aligned}
$$

where $\omega(t)=\left(1 /(p-q)^{2}\right) \int_{0}^{t}\left\{\mp(p-q) \sqrt{-K_{2} / 4 C A} H_{1}(\tau)+\right.$ $\left.\left(D(2 p-q)-E\left(E \mp \sqrt{E^{2}-4 C A}\right) p / 2 C\right) H_{2}(\tau)\right\} d \tau$.

More precisely, the obtained exact solutions and stochastic explicit solutions have rich structures which can be used to discuss the behaviors of solutions as a function of these arbitrary functions and also to provide enough freedom to build up solutions that may correspond to some particular physical situations. Moreover, it is observed that the obtained white noise functional solutions are of exponential type. It is noted that for different forms of $H_{1}(t)$ and $H_{2}(t)$, which are dependent on group velocities and Brownian effect $B(t)$, we can obtain different stochastic exact solutions of (1) from solutions (27)-(31) as in the following example. 
Example 3. Suppose that $h_{1}(t)$ and $h_{2}(t)$ are bounded or integrable functions on $\mathbb{R}_{+}$and put $H_{1}(t)=h_{1}(t)+k_{1} W(t)$ and $H_{2}(t)=h_{2}(t)+k_{2} W(t)$, where $k_{1}$ and $k_{2}$ are real constants and $W(t)$ is Gaussian white noise; that is, $W(t)=$ $\dot{B}(t) . B(t)$ is a Brownian motion. Further, taking the Hermite transformation of $H_{1}(t)$ and $H_{2}(t)$, we have $H_{1}(t, z)=h_{1}(t)+$ $k_{1} \widetilde{W}(t, z)$ and $H_{2}(t, \mathrm{z})=h_{2}(t)+k_{2} \widetilde{W}(t, \mathrm{z})$, where $\widetilde{W}(t, \mathrm{z})=$ $\sum_{k=1}^{\infty} \int_{0}^{t} \eta_{k}(s) d s z_{k}$ and $\mathrm{z}=\left(\mathrm{z}_{1}, \mathrm{z}_{2}, \ldots\right) \in \mathbb{C}^{\mathbb{N}}$ is a parameter vector and $\eta_{k}(s)$ is defined in [25]. In this case, the stochastic type solutions of (18) can express the following for (27)-(31):

$$
U_{1}(\mathbf{x}, t, \mathbf{z})=\frac{\left(K_{1}-2 D C\right) p}{E}\left[\frac{1}{p-q \exp \left\{-(p-q)\left( \pm\left(\sqrt{C A K_{1}} / 2 C A(p-q)\right) \mathbf{x}-\omega(t, \mathbf{z})\right)\right\}}\right],
$$

where $\omega(t, z)=\left(1 /(p-q)^{2}\right) \int_{0}^{t}\left\{\left(\mp\left(\sqrt{C A K_{1}} / 2 C A\right)(p \pm q)-\right.\right.$ $\left.D(p-q))\left(h_{1}(\tau)+k_{1} \widetilde{W}(t, z)\right)-\left(h_{2}(\tau)+k_{2} \widetilde{W}(t, z)\right) K_{1} q / 2 C\right\} d \tau$,

$$
U_{2}(\mathbf{x}, t, \mathbf{z})=\frac{\left(K_{2}-2 D C\right) p}{E}\left[\frac{1}{p-q \exp \left\{-(p-q)\left( \pm\left(\sqrt{C A K_{2}} / 2 C A(p-q)\right) \mathbf{x}-\omega(t, \mathbf{z})\right)\right\}}\right],
$$

where $\omega(t, z)=\left(1 /(p-q)^{2}\right) \int_{0}^{t}\left\{\left(\mp\left(\sqrt{C A K_{2}} / 2 C A\right)(p \pm q)-\right.\right.$ $\left.D(p-q))\left(h_{1}(\tau)+k_{1} \widetilde{W}(t, z)\right)-\left(h_{2}(\tau)+k_{2} \widetilde{W}(t, z)\right) K_{1} q / 2 C\right\} d \tau$,

$$
\begin{aligned}
U_{3}(\mathbf{x}, t, \mathrm{z})= & -\frac{E \mp \sqrt{E^{2}-4 C D}}{2 C}+\frac{\left(12 C D-2 E^{2}-\left(E \mp \sqrt{E^{2}-4 C D}\right)\right) p}{C E\left(2-3\left(E \mp \sqrt{E^{2}-4 C D}\right)\right)} \\
& \times\left[\frac{1}{p-q \exp \left\{-(p-q)\left( \pm(1 /(p-q)) \sqrt{-\left(E^{2}-4 C D\right) / 2 C A} \mathbf{x}-\omega(t, \mathrm{z})\right)\right\}}\right],
\end{aligned}
$$

where $\omega(t, \mathrm{z})=\left(1 / C(p-q)^{2}\right) \int_{0}^{t}\{\mp(p \quad \pm$ q) $C \sqrt{-\left(E^{2}-4 C D\right) / 2 C A}\left(h_{1}(\tau)+k_{1} \widetilde{W}(t, z)\right)+\left(2 p C D-E^{2} q+\right.$ $\left.\left.2 Q C D-(1 / 2) E\left(E-\sqrt{E^{2}-4 C D}\right)(p-q)\right)\left(h_{2}(\tau)+k_{2} \widetilde{W}(t, z)\right)\right\} d \tau$,

$$
\begin{aligned}
U_{4}(\mathbf{x}, t, \mathrm{z})= & -\frac{E \mp \sqrt{E^{2}-4 C D}}{2 C}+\frac{2\left(3 C D-E^{2}+\sqrt{E^{4} \mp 4 C E^{2} D}\right) p}{C\left(E-3 \sqrt{E^{2}-4 C D}\right)} \\
& \times\left[\frac{1}{p-q \exp \left\{-(p-q)\left( \pm(1 /(p-q)) \sqrt{-K_{1} / 4 C A} \mathbf{x}-\omega(t, z)\right)\right\}}\right],
\end{aligned}
$$

where $\omega(t, z)=\left(1 /(p-q)^{2}\right) \int_{0}^{t}\left\{\mp(p-q) \sqrt{-K_{1} / 4 C A}\left(h_{1}(\tau)+\right.\right.$ $\left.k_{1} \widetilde{W}(t, z)\right)+\left(D(2 p-q)-E\left(E \mp \sqrt{E^{2}-4 C A}\right) p / 2 C\right)\left(h_{2}(\tau)+\right.$ $\left.\left.k_{2} \widetilde{W}(t, z)\right)\right\} d \tau$, and

$$
\begin{aligned}
U_{5}(\mathbf{x}, t, \mathrm{z})= & -\frac{E \mp \sqrt{E^{2}-4 C D}}{2 C}+\frac{2\left(3 C D-E^{2}+\sqrt{E^{4} \mp 4 C E^{2} D}\right) p}{C\left(E-3 \sqrt{E^{2}-4 C D}\right)} \\
& \times\left[\frac{1}{p-q \exp \left\{-(p-q)\left( \pm(1 /(p-q)) \sqrt{-K_{2} / 4 C A} \mathbf{x}-\omega(t, \mathrm{z})\right)\right\}}\right],
\end{aligned}
$$


where $\omega(t, \mathrm{z})=\left(1 /(p-q)^{2}\right) \int_{0}^{t}\left\{\mp(p-q) \sqrt{-K_{2} / 4 C A}\left(h_{1}(\tau)+\right.\right.$ $\left.k_{1} \widetilde{W}(t, \mathrm{z})\right)+\left(D(2 p-q)-E\left(E \mp \sqrt{E^{2}-4 C A}\right) p / 2 C\right)\left(h_{2}(\tau)+\right.$ $\left.\left.k_{2} \widetilde{W}(t, \mathrm{z})\right)\right\} d \tau$.
Next, by using $\exp ^{\diamond}\{X\}=\exp \{X\}$ for nonrandom $X$ and $\int_{0}^{t} \widetilde{W}(s) d s=B(t)-(1 / 2) t^{2}$, we can obtain new versions of exact solutions for solutions (27)-(31) as follows:

$$
U_{1}(\mathbf{x}, t)=\frac{\left(K_{1}-2 D C\right) p}{E}\left[\frac{1}{p-q \exp \left\{-(p-q)\left( \pm\left(\sqrt{C A K_{1}} / 2 C A(p-q)\right) \mathbf{x}-\omega(t)\right)\right\}}\right]
$$

where $\omega(t)=\left(1 /(p-q)^{2}\right)\left(\mp\left(\sqrt{C A K_{1}} / 2 C A\right)(p \pm q)-\right.$ $(p-q) D)\left\{\int_{0}^{t} h_{1}(\tau) d \tau+k_{1}\left(B(t)-(1 / 2) t^{2}\right)\right\}+(1 /(p-$ $\left.q)^{2}\right)\left(K_{1} q / 2 C\right)\left\{\int_{0}^{t} h_{2}(\tau) d \tau+k_{2}\left(B(t)-(1 / 2) t^{2}\right)\right\}$,

$$
U_{2}(\mathbf{x}, t)=\frac{\left(K_{2}-2 D C\right) p}{E}\left[\frac{1}{p-q \exp \left\{-(p-q)\left( \pm\left(\sqrt{C A K_{2}} / 2 C A(p-q)\right) \mathbf{x}-\omega(t)\right)\right\}}\right]
$$

where $\omega(t)=\left(1 /(p-q)^{2}\right)\left(\mp\left(\sqrt{C A K_{2}} / 2 C A\right)(p \pm q)-\right.$ $(p-q) D)\left\{\int_{0}^{t} h_{1}(\tau) d \tau+k_{1}\left(B(t)-(1 / 2) t^{2}\right)\right\}+(1 /(p-$ $\left.q)^{2}\right)\left(K_{2} q / 2 C\right)\left\{\int_{0}^{t} h_{2}(\tau) d \tau+k_{2}\left(B(t)-(1 / 2) t^{2}\right)\right\}$,

$$
\begin{aligned}
U_{3}(\mathbf{x}, t)= & -\frac{E \mp \sqrt{E^{2}-4 C D}}{2 C}+\frac{\left(12 C D-2 E^{2}-\left(E \mp \sqrt{E^{2}-4 C D}\right)\right) p}{C E\left(2-3\left(E \mp \sqrt{E^{2}-4 C D}\right)\right)} \\
& \times\left[\frac{1}{p-q \exp \left\{-(p-q)\left( \pm(1 /(p-q)) \sqrt{-\left(E^{2}-4 C D\right) / 2 C A} \mathbf{x}-\omega(t)\right)\right\}}\right],
\end{aligned}
$$

where $\omega(t) \quad=\quad\left(1 / C(p-q)^{2}\right)\left(\mp\left(p \quad \pm \quad\left(1 / C(p-q)^{2}\right)\left(2 p C D-E^{2} q+2 q C D-(1 / 2) E(E-\right.\right.\right.$ q) $\left.\left.\left.C \sqrt{-\left(E^{2}-4 C D\right) / 2 C A}\right)\left\{\int_{0}^{t} h_{1}(\tau) d \tau+k_{1}\left(B(t)-(1 / 2) t^{2}\right)\right\}+\quad \sqrt{E^{2}-4 C D}\right)(p-q)\right)\left\{\int_{0}^{t} h_{2}(\tau) d \tau+k_{2}\left(B(t)-(1 / 2) t^{2}\right)\right\}$,

$$
\begin{aligned}
U_{4}(\mathbf{x}, t)= & -\frac{E \mp \sqrt{E^{2}-4 C D}}{2 C}+\frac{2\left(3 C D-E^{2}+\sqrt{E^{4} \mp 4 C E^{2} D}\right) p}{C\left(E-3 \sqrt{E^{2}-4 C D}\right)} \\
& \times\left[\frac{1}{p-q \exp \left\{-(p-q)\left( \pm(1 /(p-q)) \sqrt{-K_{1} / 4 C A} \mathbf{x}-\omega(t)\right)\right\}}\right],
\end{aligned}
$$

where $\omega(t)=\mp(1 /(p-q)) \sqrt{-K_{1} / 4 C A}\left\{\int_{0}^{t} h_{1}(\tau) d \tau+k_{1}(B(t)-\right.$ $\left.\left.(1 / 2) t^{2}\right)\right\}+\left(E\left(E \pm \sqrt{E^{2}-4 C A}\right) p / 2 C(p-q)^{2}-D(2 p-q) /(p-\right.$ $\left.q)^{2}\right)\left\{\int_{0}^{t} h_{2}(\tau) d \tau+k_{2}\left(B(t)-(1 / 2) t^{2}\right)\right\}$, and

$$
\begin{aligned}
U_{5}(\mathbf{x}, t)= & -\frac{E \mp \sqrt{E^{2}-4 C D}}{2 C}+\frac{2\left(3 C D-E^{2}+\sqrt{E^{4} \mp 4 C E^{2} D}\right) p}{C\left(E-3 \sqrt{E^{2}-4 C D}\right)} \\
& \times\left[\frac{1}{p-q \exp \left\{-(p-q)\left( \pm(1 /(p-q)) \sqrt{-K_{2} / 4 C A} \mathbf{x}-\omega(t)\right)\right\}}\right],
\end{aligned}
$$




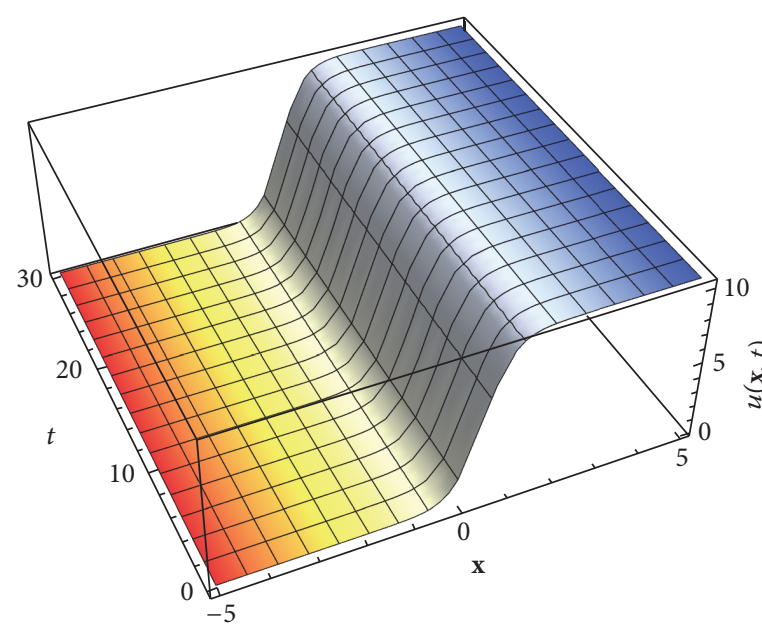

(a)

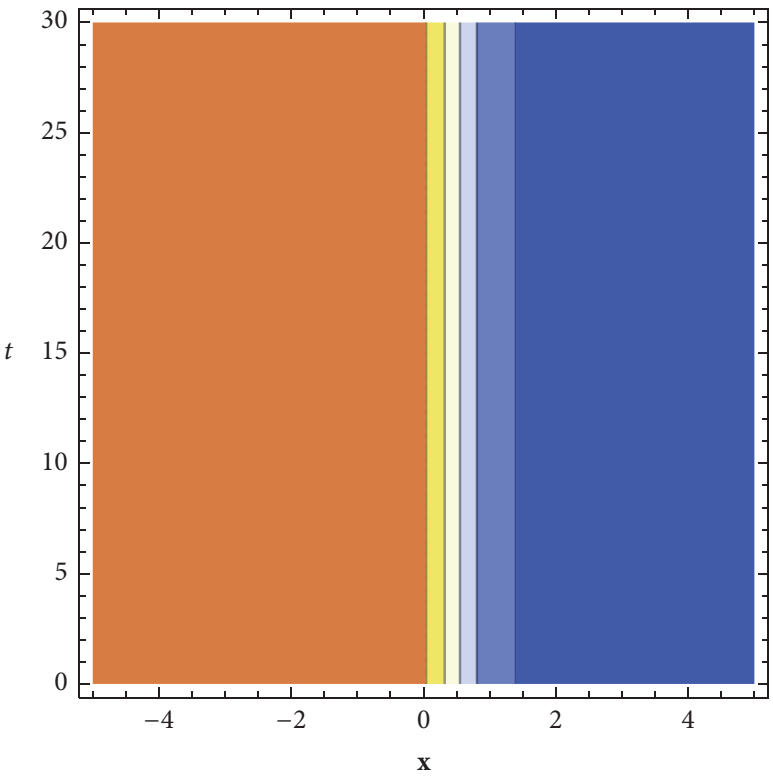

(c)

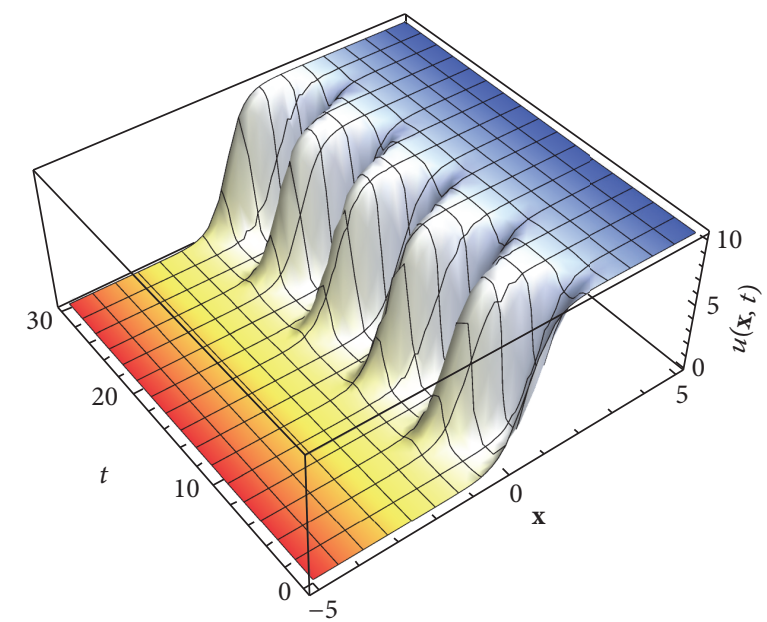

(b)

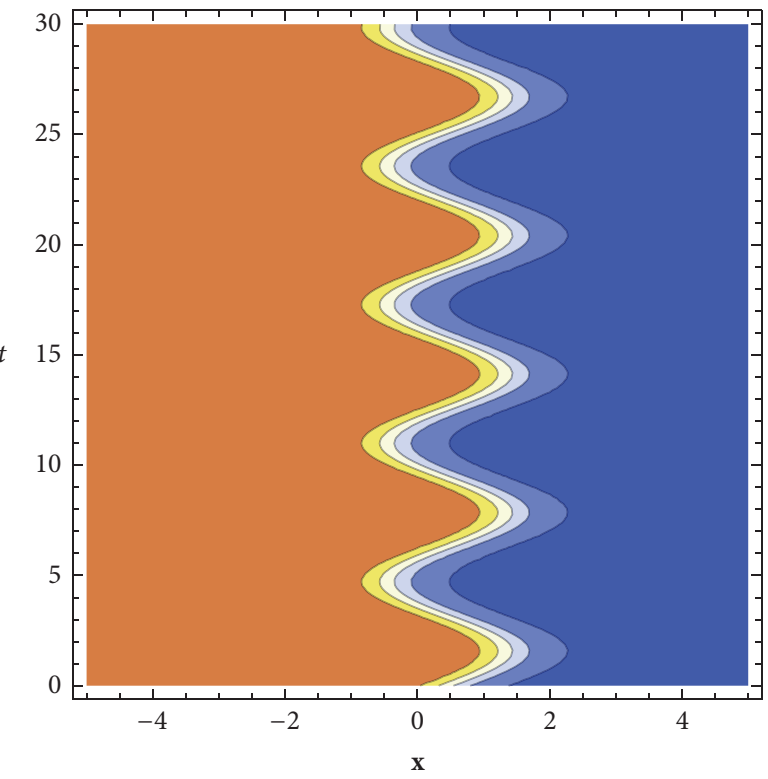

(d)

Figure 1: Profiles of the exact solution (37): (a) kink traveling wave solution without white noise term $B(t)=0$, (b) kinks traveling wave solution with $B(t)=\sin (t)$, (c) $2 \mathrm{D}$ contour of (a), and (d) $2 \mathrm{D}$ contour of (b).

where $\omega(t)=\mp(1 /(p-q)) \sqrt{-K_{2} / 4 C A}\left\{\int_{0}^{t} h_{1}(\tau) d \tau+k_{1}(B(t)-\right.$ $\left.\left.(1 / 2) t^{2}\right)\right\}+\left(E\left(E \pm \sqrt{E^{2}-4 C A}\right) p / 2 C(p-q)^{2}-D(2 p-q) /(p-\right.$ $\left.q)^{2}\right)\left\{\int_{0}^{t} h_{2}(\tau) d \tau+k_{2}\left(B(t)-(1 / 2) t^{2}\right)\right\}$.

Solutions (37)-(41) are obtained by the system technique and these solutions contain various pulse widths and group velocities for explaining the physical phenomena. In particular, we discuss the behaviors of solutions (37) and (40) with various parameter values and arbitrary functions and Brownian motions in Figures 1-4. Solution (37) indicates that pulse width is constant as $\pm \sqrt{C A K_{1}} /(2 C A(p-q))$, which are dependent on arbitrary values of parameters $A, C, D$, and $E$ with some relations and $K_{1}=2 C D+E^{2}+\sqrt{E^{4}-4 C E^{2} D}$, and group velocity $\omega(t)$ is dependent on arbitrary functions $h_{1}(t)$, $h_{2}(t)$, and Brownian motion $B(t)[26,27]$.

In Figure 1, solution (37) shows graphically the behaviors of kink traveling wave solution (37): (a) kink traveling wave solution with white noise term $B(t)=0$ and (b) kink traveling wave solitons-like solution with $B(t)=\sin (t)$ under two functions $h_{1}(t)=t, h_{2}(t)=t$, and proper values of physical parameters $A=-1, C=-1, D=1, E=5, k_{1}=1, k_{2}=1$, $p=1$, and $q=-5$ with $D=-C$ and is similar to the ChafeeInfante equation. In particular, the behaviors of (37) with $B(t)=\sin (t)$ have soft traveling waves as a big real constant $E$ is chosen. Also we represent two contours of motions (a) and (b), respectively. 


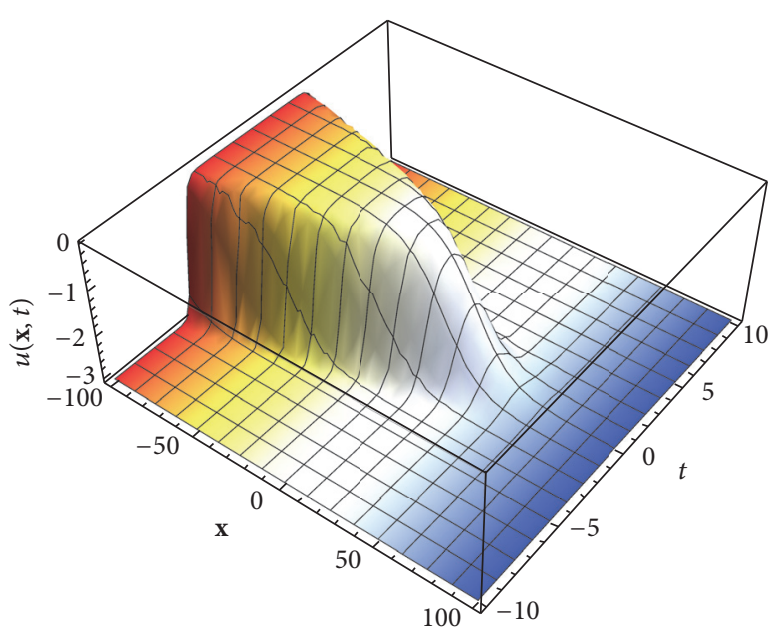

(a)

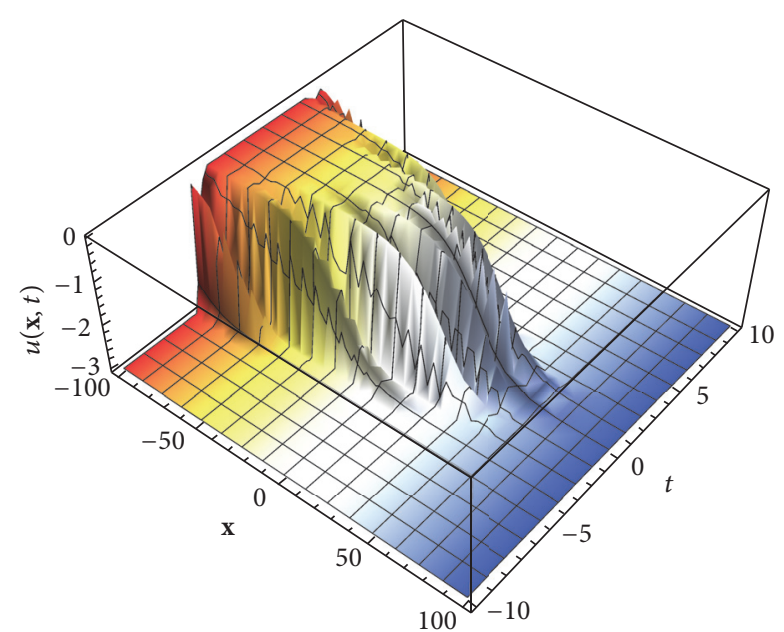

(b)

Figure 2: Profiles of the exact solution (37): (a) soliton solution with $B(t)=0$ and (b) solitons solution with $B(t)=2 \sin (5 t)$.

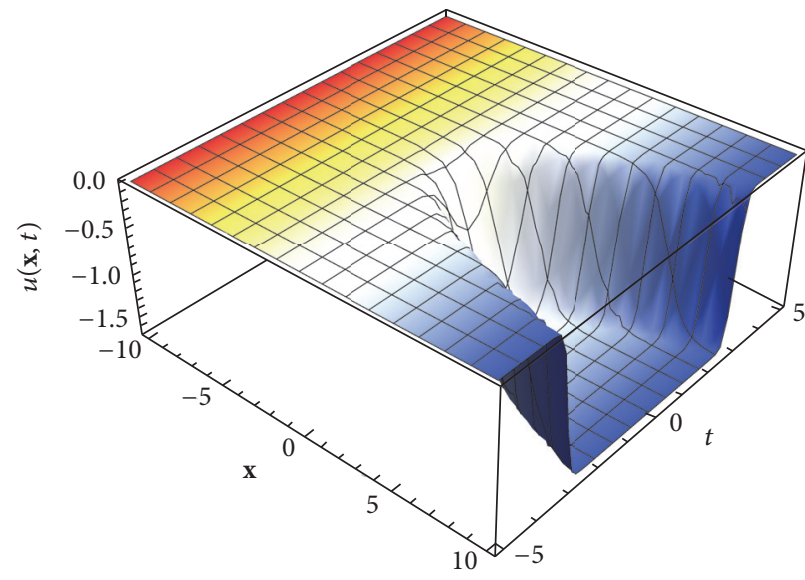

(a)

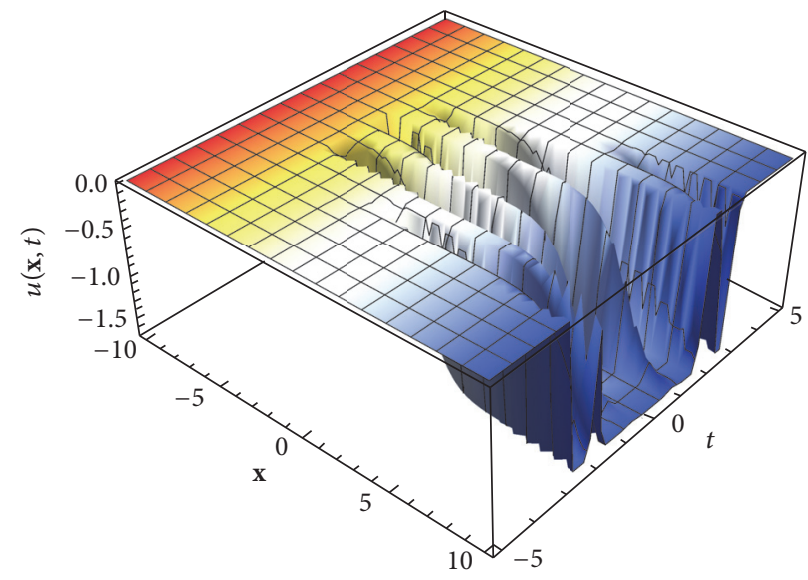

(b)

Figure 3: Profiles of the exact solution (37): (a) dark-soliton solution with $B(t)=0$ and (b) dark-solitons solution with $B(t)=2 \sin (5 t)$.

In Figure 2, solution (37) shows graphically the behaviors of soliton solution (37): (a) soliton solution with white noise term $B(t)=0$ and (b) solitons solution with $B(t)=2 \sin (5 t)$ under two functions $h_{1}(t)=-1 / 2, h_{2}(t)=0$ and proper values of physical parameters $A=2, C=3, D=-0.36$, $E=-0.8, k_{1}=5, k_{2}=0.1, p=-0.1$, and $q=1.5$ with $D=E^{2}-1,-1<E<0$.

In Figure 3, assuming two functions are $h_{1}(t)=-1 / 2$ and $h_{2}(t)=0$ and proper parameters $A=2, C=0.08, E=-0.8$, $D=-0.36, k_{1}=1, k_{2}=1, p=-1$, and $q=1.5$ with $D=E^{2}-1,-1<E<0$, we can derive the motions of solution (37) that is like a solution of Fitzhugh-Nagumo equation; Figure 3(a) represents the process of the well-known dark soliton into the flat-bottom dark soliton with white noise term $B(t)=0$ and Figure 3(b) represents the stochastic effect with deep concavity as multiple kinks solution with white noise term $B(t)=2 \sin (5 t)$.

Solution (40) indicates that pulse width is constant $\pm \sqrt{-K_{1} / 4 C A} /(p-q)$, which are dependent on arbitrary parameter values $A, C, D, E$, and $K_{1}=2 C D+E^{2}+$ $\sqrt{E^{4}-4 C E^{2} D}$, and group velocity $\omega(t)$ is dependent on arbitrary functions $h_{1}(t), h_{2}(t)$, and Brownian motion $B(t)$. In Figure 4, assuming two functions are $h_{1}(t)=t^{3}$ and $h_{2}(t)=0$ and proper parameters $A=0.1, C=-0.01, E=-0.8$, $D=-0.36, k_{1}=5, k_{2}=0.1, p=-0.1$, and $q=1.5$ with $D=E^{2}-1,-1<E<0$, we can derive the soliton-like motions of solution (40): Figure 4(a) represents the symmetric motion of two solitons solutions with white noise term $B(t)=0$ and Figure 4(b) represents the stochastic effect as nonsymmetric solitons solution with white noise term $B(t)=0.5 \sin (2 t)$.

\section{Conclusion}

In this work, we have obtained new various explicit solutions of the Wick-type stochastic reaction-Duffing equation with the Hermite transformation and the white noise theory. These results can be useful for explaining some physical problems. 


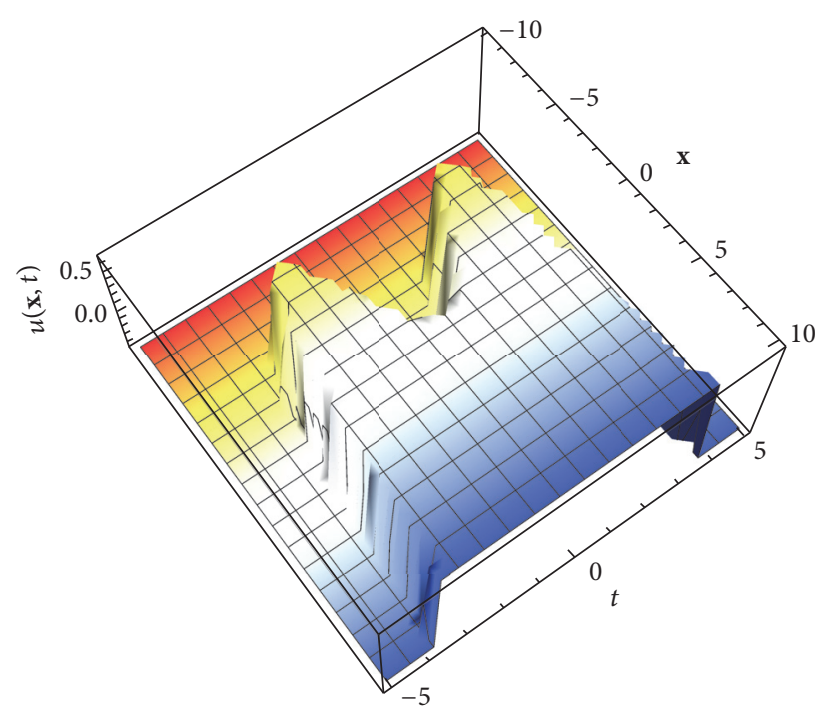

(a)

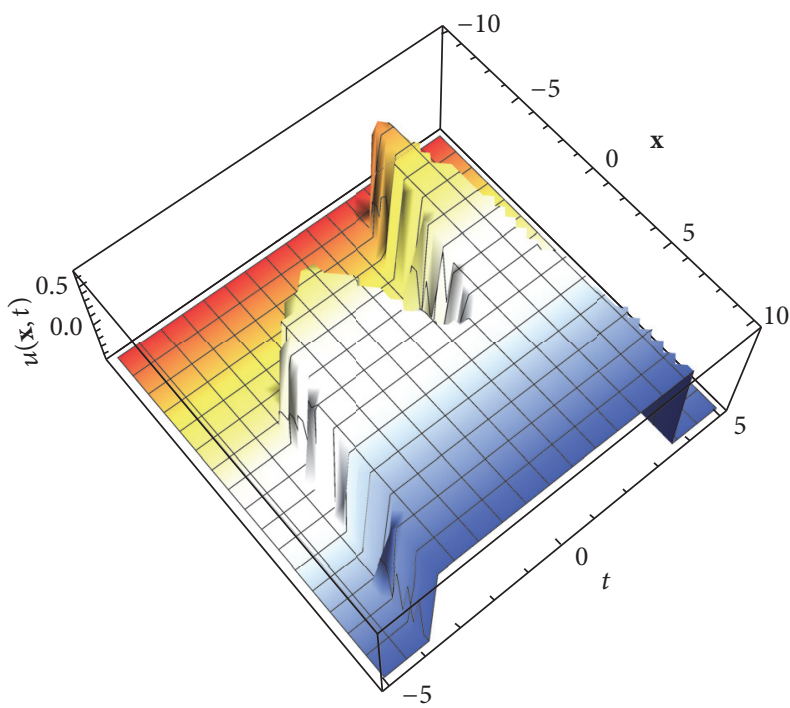

(b)

FIGURE 4: Profiles of the exact solution (40): (a) soliton solution with $B(t)=0$ and (b) solitons solution with $B(t)=0.5 \sin (2 t)$.

The system technique has been successfully applied to find more explicit solutions of the Wick-type stochastic reactionDuffing equation. From the obtained solutions, it is noted that if we take particular values for physical parameters then these solutions provide us with more analysis of new exact solutions than other existing methods. For proper values of parameters and proper functions, we show that FitzhughNagumo equation and Chafee-Infante equation are similar to the obtained solutions.

\section{Competing Interests}

The authors declare that they have no competing interests.

\section{References}

[1] S. K. Liu, Z. T. Fu, S. D. Liu, and Q. Zhao, "Jacobi elliptic function expansion method and periodic wave solutions of nonlinear wave equations," Physics Letters A, vol. 289, no. 1-2, pp. 69-74, 2001.

[2] S. Lai, X. Lv, and M. Shuai, "The Jacobi elliptic function solutions to a generalized Benjamin-Bona-Mahony equation," Mathematical and Computer Modelling, vol. 49, no. 1-2, pp. 369-378, 2009.

[3] A. Elhanbaly and M. A. Abdou, "Exact travelling wave solutions for two nonlinear evolution equations using the improved $F$ expansion method," Mathematical and Computer Modelling, vol. 46, no. 9-10, pp. 1265-1276, 2007.

[4] Sirendaoreji, "New exact travelling wave solutions for the Kawahara and modified Kawahara equations," Chaos, Solitons and Fractals, vol. 19, no. 1, pp. 147-150, 2004.

[5] A.-M. Wazwaz, "Exact solutions for the fourth order nonlinear Schrodinger equations with cubic and power law nonlinearities," Mathematical and Computer Modelling, vol. 43, no. 7-8, pp. 802-808, 2006.
[6] J. H. Choi, H. Kim, and R. Sakthivel, "Exact solution of the Wick-type stochastic fractional coupled KdV equations," Journal of Mathematical Chemistry, vol. 52, no. 10, pp. 2482-2493, 2014.

[7] M. R. Miura, Backlund Transformation, Springer, Berlin, Germany, 1978.

[8] J. Weiss, M. Tabor, and G. Carnevale, "The Painleve property for partial differential equations," Journal of Mathematical Physics, vol. 24, no. 3, pp. 522-526, 1983.

[9] M. Khalfallah, "Exact traveling wave solutions of the Boussinesq-Burgers equation," Mathematical and Computer Modelling, vol. 49, no. 3-4, pp. 666-671, 2009.

[10] A. S. Ravi Kanth and K. Aruna, "Differential transform method for solving linear and non-linear systems of partial differential equations," Physics Letters. A, vol. 372, no. 46, pp. 6896-6898, 2008.

[11] A. S. V. Ravi Kanth and K. Aruna, "Differential transform method for solving the linear and nonlinear Klein-Gordon equation," Computer Physics Communications, vol. 180, no. 5, pp. 708-711, 2009.

[12] H. A. Abdusalam and E. S. Fahmy, "Traveling wave solutions for nonlinear wave equation with dissipation and nonlinear transport term through factorizations," International Journal of Computational Methods, vol. 4, no. 4, pp. 645-651, 2007.

[13] H. Triki and A.-M. Wazwaz, "Dark solitons for a generalized Korteweg-de Vries equation with time-dependent coefficients," Zeitschrift für Naturforschung, Section A, vol. 66, no. 3-4, pp. 199-204, 2011.

[14] Q. Wang, Y. Chen, and Z. Hongqing, "A new Jacobi elliptic function rational expansion method and its application to $(1+$ 1)-dimensional dispersive long wave equation," Chaos, Solitons and Fractals, vol. 23, no. 2, pp. 477-483, 2005.

[15] W. Malfliet and W. Hereman, "The tanh method. I. Exact solutions of nonlinear evolution and wave equations," Physica Scripta, vol. 54, no. 6, pp. 563-568, 1996. 
[16] A. Biswas, "Solitary wave solution for the generalized Kawahara equation," Applied Mathematics Letters, vol. 22, no. 2, pp. 208210, 2009.

[17] H. Kim and R. Sakthivel, "New travelling wave solutions for nonlinear stochastic evolution equations," Pramana-Journal of Physics, vol. 80, no. 6, pp. 917-931, 2013.

[18] H. Kim and R. Sakthivel, "Travelling wave solutions for timedelayed nonlinear evolution equations," Applied Mathematics Letters, vol. 23, no. 5, pp. 527-532, 2010.

[19] N. A. Kudryashov, "Simplest equation method to look for exact solutions of nonlinear differential equations," Chaos, Solitons and Fractals, vol. 24, no. 5, pp. 1217-1231, 2005.

[20] H. Kim, J.-H. Bae, and R. Sakthivel, "Exact travelling wave solutions of two important nonlinear partial differential equations," Zeitschrift fur Naturforschung-Section A Journal of Physical Sciences, vol. 69, no. 3-4, pp. 155-162, 2014.

[21] S. M. Ege and E. Misirli, "The modified Kudryashov method for solving some fractional-order nonlinear equations," Advances in Difference Equations, vol. 135, no. 1, 2014.

[22] M. M. Kabir and A. Khajeh, "New explicit solutions for the Vakhnenko and a generalized form of the nonlinear heat conduction equations via Exp-function method," International Journal of Nonlinear Sciences and Numerical Simulation, vol. 10, no. 10, pp. 1307-1318, 2009.

[23] H. Holden, B. Øksendal, J. Uboe, and T. Zhang, Stochastic Partial Differential Equations, Birkhäuser, Boston, Mass, USA, 1996.

[24] H. A. Ghany, A. S. Okb El Bab, A. M. Zabel, and A.-A. Hyder, "The fractional coupled KdV equations: exact solutions and white noise functional approach," Chinese Physics B, vol. 22, no. 8, Article ID 080501, 2013.

[25] Y. Xie, "Positonic solutions for Wick-type stochastic KdV equations," Chaos, Solitons \& Fractals, vol. 20, no. 2, pp. 337-342, 2004.

[26] Y. Chen, Z. Yan, and H. Zhang, "Exact solutions or a family of variale-coefficient reaction-duffing equations via the Backlund transformation," Theoretical and Mathematical Physics, vol. 132, no. 1, pp. 970-975, 2002.

[27] C.-Q. Dai and Y.-J. Xu, "Exact solutions for a Wick-type stochastic reaction Duffing equation," Applied Mathematical Modelling, vol. 39, no. 23-24, pp. 7420-7426, 2015. 


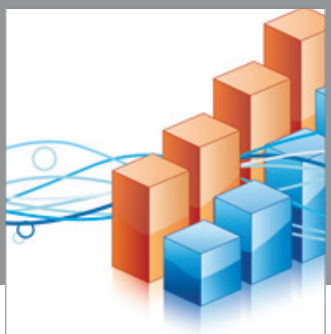

Advances in

Operations Research

vatem alat4

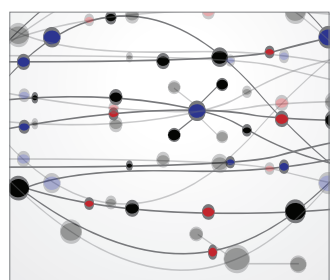

\section{The Scientific} World Journal
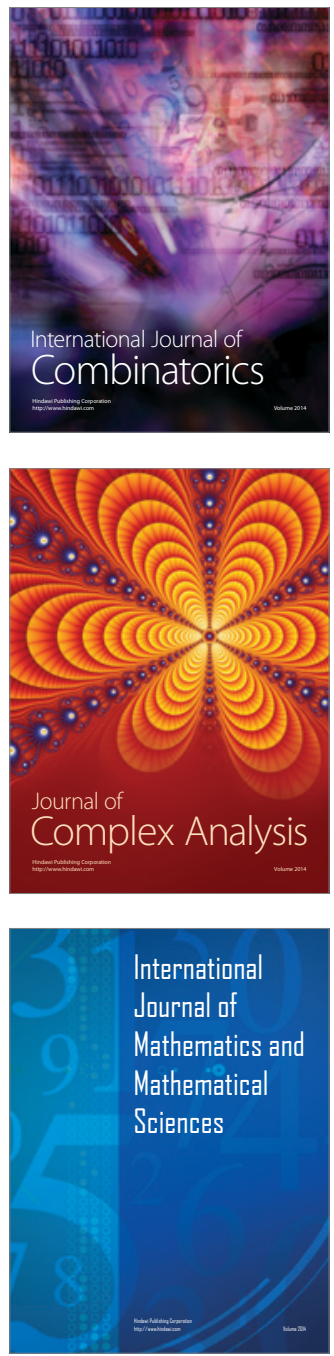
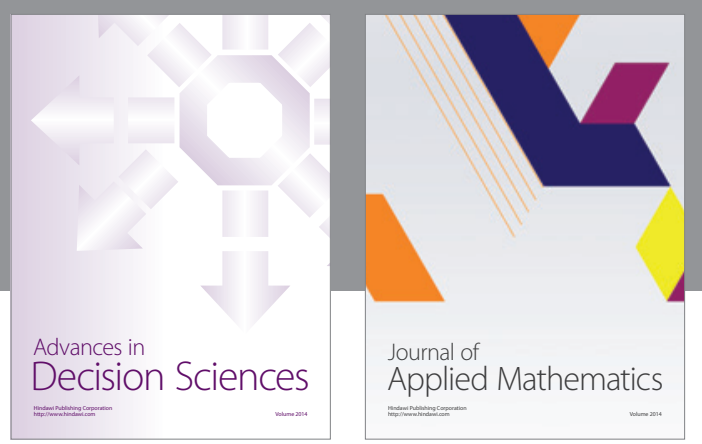

Algebra

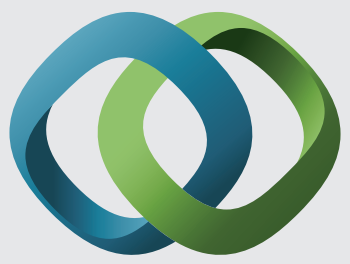

\section{Hindawi}

Submit your manuscripts at

http://www.hindawi.com
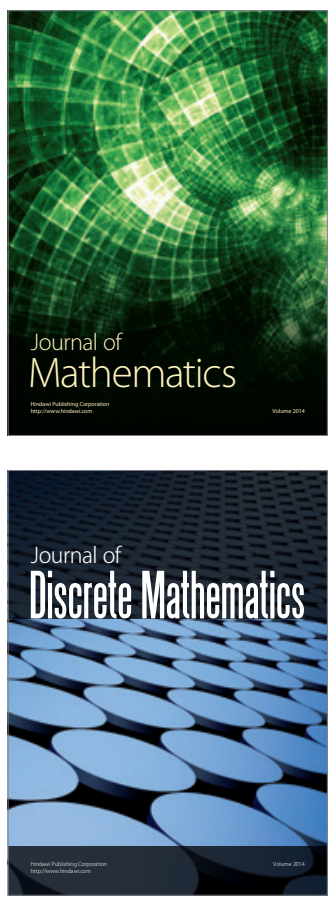

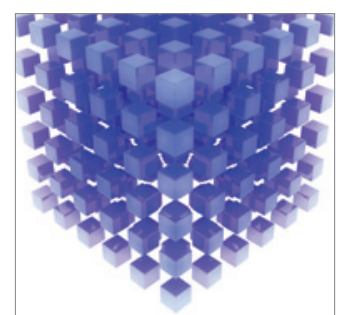

Mathematical Problems in Engineering
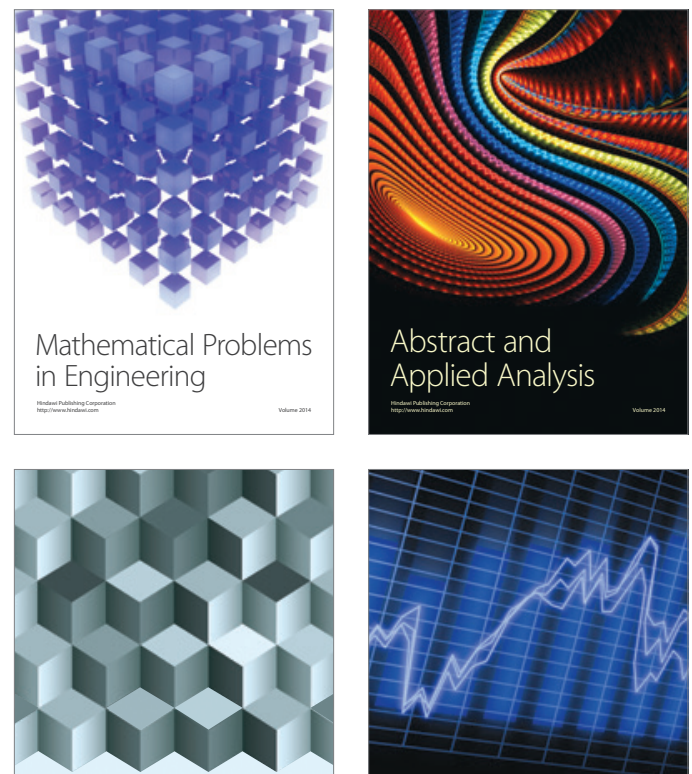

Journal of

Function Spaces

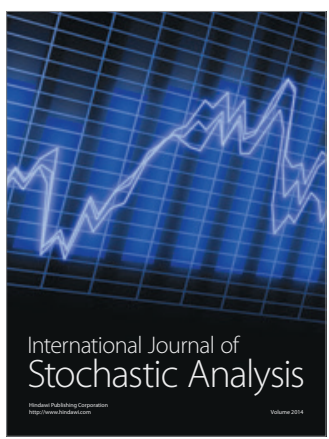

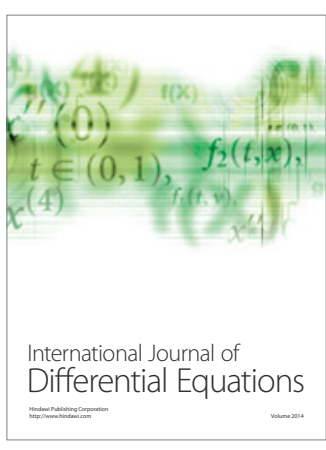
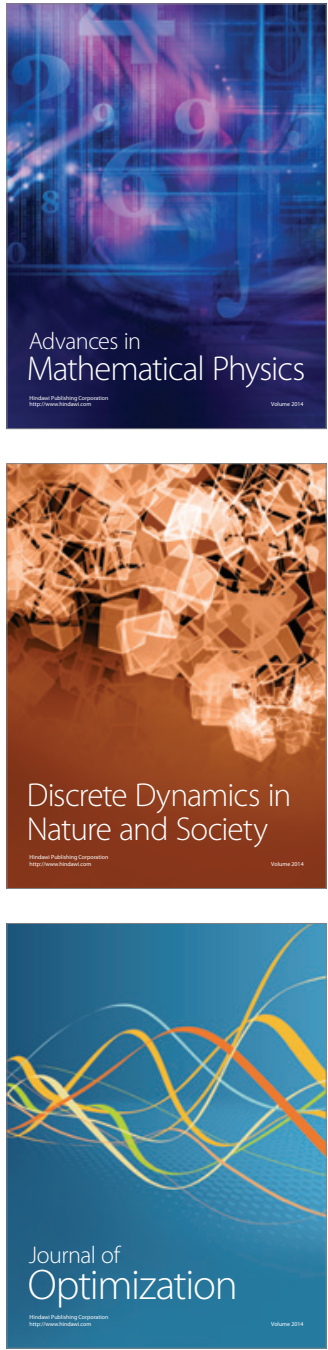